Revista Gestionar

Vol. 1 Núm. 2 (2021)

ISSN: $2810-8264$ ISSN-L: $2810-823 \mathrm{X}$

Editada por: Instituto Universitario de Innovación Ciencia y Tecnología Inudi Perú

\title{
Politicas contables y su incidencia en la elaboración de los estados financieros en un municipio del Perú
}

Accounting policies and their impact on the preparation of financial statements in a municipality of Peru

Políticas contábeis e seu impacto na preparação das demonstrações financeiras em um município do Peru

\author{
Nadia Choquehuayta ${ }^{1}$ \\ Municipalidad Distrital de Pillpinto, Cusco \\ Nancy Martell \\ Universidad Nacional del Altiplano, Perú \\ Yoshia Martell \\ Universidad Nacional del Altiplano, Perú
}

DOI: https://doi.org/10.35622/j.rg.2021.02.003

Recibido 30/03/2021/ Aceptado 15/04/2021

\begin{abstract}
RESUMEN. El objetivo fue determinar la incidencia de la aplicación de las políticas contables para el sector público en la elaboración de estados financieros en la Municipalidad de Pillpinto. Para esto se describió las formas de aplicación de las políticas contables en la preparación y presentación de la situación legal y técnica de los activos, pasivos y patrimonio de la entidad. Se aplicó el método tipo descriptivo analítico, mediante el análisis de estados financieros y descripción a través de una encuesta del conocimiento de las politicas contables al personal que trabaja en la entidad. Se aprecia que la incidencia de la aplicación de las normas internacionales de contabilidad para el sector público (NICSP) y demás políticas contables en la elaboración de los estados financieros de la municipalidad es deficiente. El 49\% de los profesionales no aplicaron adecuadamente las NICSP y demás políticas contables en la elaboración de la información contable porque el $56 \%$ de los servidores públicos no tienen conocimiento de dichas normas. Se encontró que la aplicación de las normas internacionales de contabilidad para el sector público en la elaboración de los estados financieros. Por lo tanto, la información que presentan estos documentos es deficiente para la toma de decisiones, ya que no cuentan con las características cualitativas que se requieren. Para resarcir esta falencia se debe aplicar capacitaciones, evaluaciones y las sanciones correspondientes a los profesionales a cargo del proceso contable. Si se busca garantizar la aplicación de las politicas contables de manera correcta y oportuna se debe implantar el control interno en las unidades que manejan la información contable.
\end{abstract}

PALABRAS CLAVE: Estados financieros, normas internacionales, sector público, políticas contables.

\footnotetext{
${ }^{1}$ Correspondencia: ilujanom@est.unap.edu.pe
} 
ABSTRACT. The objective was to determine the incidence of applying accounting policies for the public sector in the preparation of financial statements in the Municipality of Pillpinto. For this, the forms of application of accounting policies in the preparation and presentation of the entity's legal and technical situation of the assets, liabilities, and equity were described. The analytical descriptive type method was applied through the analysis of financial statements and description through a survey of the knowledge of the accounting policies to the personnel working in the entity. It is appreciated that the incidence of the application of international accounting standards for the public sector (IPSAS) and other accounting policies in the preparation of the municipality's financial statements is deficient. $49 \%$ of the professionals did not correctly apply the IPSAS and other accounting policies in the practice of the accounting information because $56 \%$ of the public servants are not aware of these standards. It was found that the application of international accounting standards for the public sector in the preparation of financial statements. Therefore, the information presented in these documents is deficient for decision-making since they do not have the required qualitative characteristics. Training, evaluations, and corresponding sanctions must be applied to the professionals in charge of the accounting process to compensate for this deficiency. If the aim is to guarantee the application of accounting policies in a correct and timely manner, internal control must be implemented in the units that handle accounting information.

KEYWORDS: Financial statements, international standards, public sector, accounting policies.

RESUMO. O objetivo foi determinar a incidência da aplicação das políticas contabilísticas do setor público na preparação das demonstrações financeiras do Municipio de Pillpinto. Para tal, foram descritas as formas de aplicação das políticas contabilísticas na preparação e apresentação da situação jurídica e técnica dos ativos, passivos e patrimônio líquido da entidade. Foi aplicado o método do tipo analítico descritivo, através da análise das demonstrações financeiras e da descrição através do levantamento do conhecimento das politicas contabilisticas ao pessoal que trabalha na entidade. É apreciado que a incidência da aplicação das normas internacionais de contabilidade para o setor público (IPSAS) e outras políticas contábeis na preparação das demonstrações financeiras do municipio é deficiente. 49\% dos profissionais não aplicaram adequadamente as IPSAS e outras políticas contábeis na preparação da informação contábil porque $56 \%$ dos servidores públicos desconhecem essas normas. Constatou-se a aplicação das normas internacionais de contabilidade para o setor público na preparação das demonstrações financeiras. Portanto, as informações apresentadas por esses documentos são deficientes para a tomada de decisão, uma vez que não possuem as características qualitativas exigidas. Para suprir esta deficiência, devem ser aplicados treinamentos, avaliações e respectivas sanções aos profissionais responsáveis pelo processo contábil. Se o objetivo é garantir a aplicação das políticas contabilísticas de forma correta e atempada, deve haver controlo interno nas unidades que tratam da informação contabilística.

PALAVRAS CHAVE: Demonstrações financeiras, padrões internacionais, setor público, politicas contábeis. 


\section{INTRODUCCIÓN}

Las Políticas contables son los principios, bases, acuerdos reglas y procedimientos específicos adoptados por la entidad en la elaboración y presentación de sus estados financieros (IASB, 2005). Las políticas contables que emite la dirección general de contabilidad pública están en congruencia al marco conceptual y las normas internacionales de contabilidad para el sector público, de las cuales en nuestro país están oficializadas 32 NICSP, mientras que el marco conceptual aún está pendiente por oficializar (Ministerio de Economia y Finanzas, 2013).

Los estados financieros del sector público suministran información útil y confiable de los resultados de la gestión de las entidades de la actividad gubernamental y la actividad empresarial, para la toma de decisiones (CGR, 2007).

Otros investigadores concluyeron que en "El análisis de la situación actual de las políticas contables para el sector público, utilizadas en la elaboración y presentación de los estados financieros en el año fiscal 2013, de la municipalidad distrital de El Porvenir, identificándose que no fueron formulados de acuerdo a lo normado por las NIC SP" (Gonzales \& Lopez, 2014). Asimismo, otro investigador concluyo que, " Se evaluó y determino que las Políticas Contables para el Sector Público inciden significativamente en la elaboración presentación de los estados financieros de la Municipalidad Distrital de Eten, ocasionando que no se cuente con información confiable para la toma de decisiones y por ende, se reduzca el nivel de eficiencia en la entidad." (Tapia, 2007).

Se buscó precisar y medir la aplicación que han tenido estas normas en la elaboración del conjunto de los Estados Financieros, lastimosamente se han encontrado muchas falencias por la falta de una correcta aplicación por parte de los profesionales de las áreas contables, la cual se puede corregir a través de capacitaciones, evaluaciones y sanciones al personal que elabora la información contable, así mismo se debe implantar el control interno para que de ese modo la información financiera refleje la real situación de los activos, pasivos y patrimonio de la entidad. 


\section{MÉTODO}

\section{Objetivo}

Determinar la medida en que incide la aplicación de las políticas contables en la elaboración de estados financieros en la municipalidad distrital de Pillpinto en los años 2015 al 2017.

\section{Lugar de estudio}

La presente investigación se realizó en la Municipalidad de Pillpinto; se ubica al este de la provincia de Paruro en las coordenadas $13^{\circ} 56^{\prime} 30^{\prime \prime}$ de Latitud sur y $71^{\circ} 46^{\prime} 00^{\prime \prime}$ de Longitud oeste. Cuenta con una superficie de $79.13 \mathrm{Km}^{2}$, que se emplaza a través de cuatro pisos ecológicos: Quechua Bajo, Quechua Alto, Suni y en menor medida, la Puna; en un rango altitudinal que va de los 2850 m.s.n.m. a los 4250 m.s.n.m. Pertenece en su totalidad a la cuenca del Apurímac por su vertiente izquierda; desde la cabecera de la cuenca, divisoria con el río Velille, hasta el piso del Valle del Apurímac. Está ubicado entre los distritos de Accha (Paruro) y Acos (Acomayo), a orillas del río Apurímac y en la parte baja de la quebrada Molino pampa, que tiene su cabecera en el distrito de Accha; orientándose mucho más a la provincia vecina de Acomayo, no solo en asuntos comerciales, sino inclusive en trámites administrativos.

\section{Método}

En todo estudio o investigación es de importancia fundamental que los hechos y relaciones que se establece, a partir de los resultados obtenidos o nuevos conocimientos; tengan el grado máximo de exactitud y confiabilidad. Para ello se utilizó una metodología que se siguió para establecer lo significativo de los hechos y fenómenos hacia los cuales estuvo encaminado el significado de la investigación, se utilizaron los métodos descriptivo analítico.

\section{Diseño}

El diseño de la investigación para el presente estudio es de carácter no experimental y cuyo diseño es el explicativo casual, tal como describe a más detalle (Hosford \& Bayarre, 2009). Para el presente estudio se establecerá el diseño: 
Estados financieros $=F$ (Politicas contables)

Dónde: Estados financieros es la variable dependiente, políticas contables es la variable independiente.

\section{Población y muestra}

Por las características del estudio y obtener mayores incidencias y más información se ha establecido como la población a los servidores públicos que trabajan en las unidades de contabilidad, tesorería, abastecimiento, presupuesto de la municipalidad distrital de Pillpinto de los años 2015 al 2017.

Tabla 1

Población de servidores públicos.

\begin{tabular}{ccc}
\hline Unidades o Áreas & $\begin{array}{c}\text { Número de } \\
\text { servidores } \\
\text { públicos }\end{array}$ & $\%$ \\
\hline Contabilidad & 1 & $14 \%$ \\
Tesoreria & 2 & $28 \%$ \\
Abastecimiento y logística & 3 & $43 \%$ \\
Presupuesto & 1 & $14 \%$ \\
TOTAL & 7 & $100 \%$
\end{tabular}

Fuente: Información registrada en la unidad de recursos humanos la MDP. El tipo de muestreo es no probabilístico y discrecional es decir que ha sido elegido por criterio propio. Para obtener una mayor incidencia se escogió como muestra a toda la población.

Tabla 2

Afijación proporcional al tamaño del estrato

\begin{tabular}{ccc}
\hline Rubro & $\begin{array}{c}\text { Numero de } \\
\text { documentos }\end{array}$ & $\mathrm{N}_{\mathrm{h}}$ \\
\hline Contabilidad & 1 & 1 \\
Tesorería & 2 & 2 \\
Abastecimiento y logística & 3 & 3 \\
Presupuesto & 1 & 1 \\
TOTAL & 7 & 7 (muestra) \\
\hline
\end{tabular}

Fuente: elaboración propia 


\section{RESULTADOS Y DISCUSIONES}

Se presentan los resultados obtenidos previa aplicación de los instrumentos de recolección de los datos a la población objeto de estudio. Se interpretaron los resultados con la finalidad de facilitar la comprensión de estos atendiendo a la problemática planteada, y a sus objetivos de estudio.

El diagnóstico de la aplicación de las políticas contables que aplica la municipalidad distrital de Pillpinto, fue determinado mediante la utilización del cuestionario; con el fin de orientar la investigación y servir de soporte al estudio, debido a que esta técnica de recolección de datos permitirá determinar las debilidades o puntos críticos de la entidad.

Los resultados obtenidos en la tabla 3 nos demuestran que:

1. El $57 \%$ de los servidores públicos de la municipalidad distrital de Pillpinto manifestaron que sí tienen conocimiento sobre las politicas contables, cambios en las estimaciones contables y errores, también manifiestan que en el reconocimiento y valuación de las existencias sí se aplica la NICSP $\mathrm{N}^{\circ} 12$ inventarios, asimismo si aplican la NICSP $\mathrm{N}^{\circ} 19$ en las provisiones, pasivos contingentes y activos contingentes; sin embargo el $43 \%$ de los servidores públicos encuestados no tienen conocimiento sobre las politicas contables, cambios en las estimaciones contables y errores, lo mismo sucede en el reconocimiento y valuación de las existencias que no aplican la NICSP $\mathrm{N}^{\circ} 12$ inventarios y de la misma forma tampoco aplican la NICSP $\mathrm{N}^{\circ} 19$ en las provisiones, pasivos contingentes $\mathrm{y}$ activos contingentes.

2. El $86 \%$ de los servidores públicos de la municipalidad distrital de Pillpinto manifestaron que los ingresos por impuestos municipales sí son valuados y tratados según la NICSP $n^{\circ} 23-$ ingresos de transacciones sin contraprestación, y aplican la NICSP $\mathrm{n}^{\circ} 25$ en el tratamiento de los beneficios a los empleados ; el 14\% de los servidores públicos encuestados perciben que los ingresos por impuestos municipales no son valuados $\mathrm{y}$ tratados según la NICSP $n^{\circ} 23$ - ingresos de transacciones sin 
contraprestación y no aplican la NICSP n ${ }^{\circ} 25$ en el tratamiento de los beneficios a los empleados.

Tabla 3

Conocimiento y aplicación de las normas internacionales de contabilidad para el sector público para la elaboración de los estados financieros de la municipalidad distrital de Pillpinto en los años 2015 al 2017

Items

1. ¿Tiene conocimiento de las Políticas

Contables, Cambios en las Estimaciones

Contables y Errores?

2. ¿El reconocimiento y valuación de las

existencias se aplica la NIC SP $\mathrm{N}^{\circ} 12$

Inventarios?

3. ¿Para los hechos ocurrido después de la fecha de presentación se aplica la NIC SP $\mathrm{N}^{\circ} 14$ ?

4. ¿El costo de valuación y el tratamiento de las depreciaciones según NIC SP $\mathrm{N}^{\circ} 17$ Propiedades, Planta y Equipo?

5. ¿Se aplica la NIC SP $\mathrm{N}^{\circ} 19$ en las

Provisiones, Pasivos Contingentes y Activos Contingentes?

6. ¿Se aplica la NIC SP N ${ }^{\circ} 21$ en el Deterioro del Valor de Activos No Generadores de Efectivo?

7. ¿Los ingresos por impuestos municipales son valuados y tratados según la NIC SP $\mathrm{N}^{\circ} 23$ - Ingresos de Transacciones sin Contraprestación (¿Impuestos y Transferencias?

8. ¿Tiene conocimiento de la NIC SP N ${ }^{\circ} 24-$ Presentación de información del presupuesto en los estados financieros?

9. ¿Se aplica la NIC SP $\mathrm{N}^{\circ} 25$ en el tratamiento de los Beneficios a los Empleados?

$$
\text { Total respuestas }
$$

$\begin{array}{llllll}6 & 86 \% & 1 & 14 \% & 7 & 100 \%\end{array}$

$\begin{array}{llllll}4 & 57 \% & 3 & 43 \% & 7 & 100 \%\end{array}$

$\begin{array}{llllll}1 & 14 \% & 6 & 86 \% & 7 & 100 \%\end{array}$

Total $\mathrm{Si} \quad$ No

$\begin{array}{llllll}5 & 57 \% & 3 & 43 \% & 7 & 100 \%\end{array}$

$\begin{array}{llllll}1 & 14 \% & 6 & 86 \% & 7 & 100 \%\end{array}$

$\begin{array}{llllll}1 & 14 \% & 6 & 86 \% & 7 & 100 \%\end{array}$

$\begin{array}{llllll}4 & 57 \% & 3 & 43 \% & 7 & 100 \%\end{array}$

$14 \%-6.86 \%-7.100 \%$


De la tabla 3 se aprecia que la mayoría de los conformantes de la muestra del estudio tienen conocimiento de las normas internacionales de contabilidad para el sector público, debido a las capacitaciones que el ministerio de economía y finanzas (MEF) realiza en preocupación de que los gobiernos locales y regionales alcanzan sus estados financieros con observaciones, pero hasta la fecha es poca la aplicación que se hace, en la investigación se percibió que, el desinterés de aplicar correctamente los principios contables es porque posteriormente no se evalúa los conocimientos adquiridos y no se sanciona por estas malas prácticas contables.

Por tanto, se concluye que los servidores públicos que preparan la información contable y financiera no aplican de manera correcta las normas internacionales de contabilidad para el sector público, es por tal razón que la información financiera no es fiel imagen de las transacciones que realiza la entidad, a pesar de que el MEF realiza cursos para que los servidores públicos empiecen a aplicar estas políticas contables aún no se toma las medidas preventivas y correctivas.

Los resultados obtenidos en la tabla 4 nos demuestran que:

1. El 29\% de los servidores públicos de la municipalidad distrital de Pillpinto manifiestan que sí consideran que el estado de situación financiera refleja de manera razonable los saldos contables de la entidad en los años 2015 al 2017, también manifiestan que las notas a los estados financieros de los años 2015 al 2017 sí se especifican claramente las politicas contables, estimaciones contables y errores aplicados en el estado de gestión, y que las notas a los estados financieros son elaborados y presentados correctamente; el $71 \%$ de los servidores públicos perciben que no consideran que el estado de situación financiera refleja de manera razonable los saldos contables de la entidad en los años 2015 al 2017, también manifestaron que las notas a los estados financieros de los años 2015 al 2017 no se especificaron claramente las politicas contables, estimaciones contables y errores aplicados en el estado de gestión, y además que las notas a los estados financieros no son elaborados y ni presentados correctamente. 
2. El $86 \%$ de los servidores públicos encuestados de la municipalidad distrital de Pillpinto manifestaron que sí creen que fue necesario aplicar politicas contables, estimaciones contables y errores para la elaboración y presentación del estado de cambios en el patrimonio neto, en los años 2015 al 2017; el 14\% de los servidores públicos perciben que no creen que fue necesario aplicar politicas contables, estimaciones contables y errores para la elaboración y presentación del estado de cambios en el patrimonio neto, en los años 2015 al 2017.

\section{Tabla 4}

Conocimiento y aplicación de las políticas contables en el conjunto de estados de situación financiera de la municipalidad distrital de Pillpinto en los años 2015 al 2017

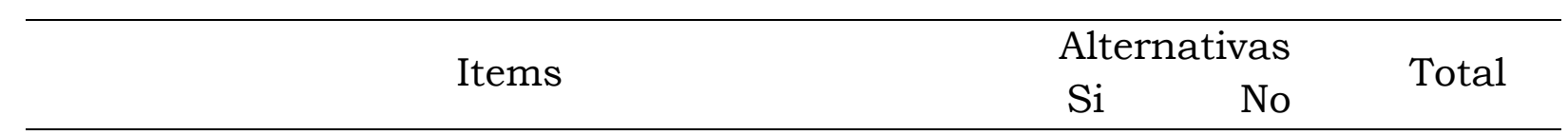

1. ¿Cree que el Estado de Situación Financiera refleja de manera razonable los saldos contables de la entidad en los años 2015 al 2017 ?

$\begin{array}{llllll}2 & 29 \% & 5 & 71 \% & 7 & 100 \%\end{array}$

2. ¿En las notas a los Estados Financieros de los años 2015 al 2017 se especifica claramente las politicas contables, estimaciones contables y errores aplicados en el estado de gestión?

$\begin{array}{llllll}2 & 29 \% & 5 & 71 \% & 7 & 100 \%\end{array}$

3. ¿Cree que fue necesario aplicar politicas contables, estimaciones contables y errores para la elaboración y presentación del estado de cambios en el patrimonio neto, en los años 2015 al 2017?

$\begin{array}{llllll}6 & 86 \% & 1 & 14 \% & 7 & 100 \%\end{array}$

4. ¿La información presentada en el estado de flujos de efectivo ha sido desarrollada en base a politicas contables, estimaciones contables y errores?

5. ¿Cree usted que las notas a los Estados Financieros son elaborados y presentados correctamente?

Total respuestas

$\begin{array}{llllll}2 & 29 \% & 5 & 71 \% & 7 & 100 \%\end{array}$ $\begin{array}{lllllll}18 & 51 \% & 17 & 49 \% & 35 & 100 \%\end{array}$

Fuente: Encuesta realizada a los servidores públicos que trabajan en las áreas que elaboran información contable 
De la tabla 4 se aprecia que la mayoría considera que los estados financieros y las notas a los estados financieros no reflejan de manera razonable y no se han presentado de manera correcta, también consideran que no es necesario aplicar políticas contables, estimaciones contables y errores en todo el conjunto de los estados financieros y todas las demás políticas contables para obtener una información de calidad.

\section{DISCUSIONES}

Es deficiente la aplicación de las políticas contables para el reconocimiento, valuación y tratamiento de la información contable, coincidimos con las conclusiones de otro investigador que indican que: "Se observó e identificó que los profesionales de las áreas administrativas de la Municipalidad Distrital de Eten no conocen las políticas contables que deben de utilizar para el tratamiento de las operaciones contables, por ende no las aplican en la formulación de sus estados financieros incumpliendo con lo establecido en las Normas Internacionales de Contabilidad para el Sector Público; y los lineamientos dictados por Dirección General de Contabilidad Pública del Ministerio de Economía y Finanzas" (Tapia, 2007), Podemos afirmar que esto se da por la falta de un sistema de control interno y la no aplicación de sanciones al personal que trabaja en las áreas de administración, contabilidad tesorería, logística, presupuesto e incluso subgerencia de infraestructura.

Un factor condicionante y limitante para el desarrollo de la investigación fue el acceso a la información, pues no todas las áreas han colaborado, indicando que por el cambio de gestión la documentación está incompleta. De ahí consideramos que, si se va a realizar un proceso de regularización, este va a encontrarse con la misma limitante y condicionante, pues sin documentos sustentatorios es difícil regularizar la información de ejercicios anteriores, otra limitante que se observo es el bajo presupuesto que tiene la municipalidad lo que no ha permitido contratar personal especializado.

Según lo hallado en la presente investigación, se han identificado las políticas contables para el sector público necesarias para la elaboración y presentación de los estados financieros, las mismas que ayudaran a mejorar el desempeño organizacional en la entidad, a la vez los estados financieros 
son informes financieros que pretenden satisfacer las necesidades de información de los usuarios que no tienen capacidad de requerir la preparación de informes financieros concretos para satisfacer sus necesidades de información específicas (IPSASB, 2013); por lo consiguiente, será necesario que la información económica-financiera sea fiel imagen del activo, pasivo y patrimonio de la entidad, explicando con veracidad e indicando su influencia sobre el patrimonio, la situación financiera y los resultados de la entidad.

Previo al desarrollo del estudio, se tuvo acceso a los estados financieros de la entidad de los periodos 2015 al 2017, donde se pudo observar que en las notas a los estados financieros, se establecen varias políticas contables, pero estas no se han utilizado en la elaboración y presentación de los mismos, estas notas iniciales deben identificar a la municipalidad distrital de Pillpinto, resumir sus políticas y prácticas contables y los asuntos de importancia relativa. Las notas deben ser presentadas en una secuencia lógica, guardando en cuanto sea posible el mismo orden de los rubros de los estados financieros.

La aplicación de políticas contables es la mejor opción para perfeccionar la elaboración y presentación de estados financieros, por lo que se ha analizado el estado de situación financiera, estado de gestión, estado de cambios en el patrimonio neto y el estado de flujos de efectivo de los años 2015 al 2017, en la que se verifico que los rubros contables no reflejan el saldo real.

Por otro lado, se debe mencionar que la municipalidad distrital de Pillpinto, en los años 2015 al 2017 indica que solo aplicó la política contable del devengo, por lo tanto no cumple en su totalidad con los criterios establecidos por las NICSP $\mathrm{N}^{\circ}$ 01: Presentación de estados financiero, la NICSP $\mathrm{N}^{\circ} 03$ : Políticas contables, cambios en las estimaciones contables y errores, y todas aquellas NICSP relacionadas según IPSAS, (2017) para la elaboración y presentación en los estados financieros. 


\section{CONCLUSIONES}

En conclusión la información que se presentó en los estados financieros en los años 2015 al 2017 no son razonables porque no reflejan los verdaderos saldos contables, por tanto urge que se haga un proceso de análisis de cuentas, inventarios y valuaciones de los activos de la entidad, conciliaciones bancarias, liquidaciones de obras, para hacer los ajustes de corrección a los errores encontrados, para esto los funcionarios de la entidad tienen que ver la importancia de realizar este proceso, mientras no se haga todas estas actividades, la información de años venideros y del presente se seguirá presentando con esas falencias.

Es necesario que el personal que trabajan en las unidades que procesan información contable debe ser capacitado y evaluado, y se debe implantar un sistema de control interno en la entidad para que cada área que tiene que ver con la elaboración de información contable aplique las políticas contables y que se establezcan directivas para determinar responsabilidades si estas no se cumplen.

\section{REFERENCIAS}

CGR. (2007). 2 . Información Financiera, 112-157. Retrieved from https://www.mef.gob.pe/contenidos/conta_publ/2007/tomo1/ 2_INFORMACION_FINANCIERA.pdf

Gonzales, C., \& Lopez, K. (2014). Las politicas contables del Sector público en la elaboración y presentación de los Estados Financieros de la Municipalidad Distrital de El Porvenir. Retrieved from http://repositorio.upao.edu.pe/bitstream/upaorep/341/1/GON ZALEZ_CLAUDIA_POLITICAS_CONTABLES_ESTADOS_FINANCI EROS.pdf

Hosford, R., \& Bayarre, H. (2009). Parte II. Metodología de la Investigación Científica 1. Métodos Y Técnicas Aplicadas a La Investigación En Atención Primaria de Salud, 53. Retrieved from http:/ / files.sld.cu/isss / files /2009/02/curso-metodologia.pdf 
IASB. (2005). Norma Internacional de Contabilidad n o 8 ( NIC 8 ) Políticas contables, cambios en las estimaciones contables y errores Norma Internacional de Contabilidad no 8 ( NIC 8 ) Políticas contables, cambios en las estimaciones contables y errores Objetivo Alc, 8(Nic 8), 1-11. Retrieved from https://www.mef.gob.pe/contenidos/conta_publ/con_nor_co/vi gentes/nic/8_NIC.pdf

IPSAS. (2017). Las NICSP en su bolsillo, 56. Retrieved from https://www2.deloitte.com/content/dam/Deloitte/pe/Documen ts/audit/NICSP en su bolsillo 2017.pdf

IPSASB. (2013). El marco conceptual para la información financiera con propósito general de las entidades del sector público, 2013. Retrieved from https:/ / www.ifac.org/system/files / publications / files /A9IPSASB-Conceptual-Framework_0.pdf

Tapia, L. (2007). Las Politicas Contables del Sector Publico y su incidencia en los Estados Financieros de la Municipalidad Distrital de Eten, 2015. Retrieved from http://repositorio.ucv.edu.pe/bitstream/handle/UCV/10192/t apia_dl.pdf?sequence $=1$ 